Check for updates

Cite this: RSC Adv., 2020, 10, 23233

Received 18th January 2020

Accepted 17th April 2020

DOI: $10.1039 / d 0 r a 00537 a$

rsc.li/rsc-advances

\title{
An optical-magnetic Material as a toxic gas filter and sensing device
}

\author{
Thuanny Almeida Moraes, (D) a Maria Julia Farrôco, ${ }^{a}$ Ketly Pontes, ${ }^{b}$ Magda Fontes \\ Bittencourt, ${ }^{\mathrm{C}}$ Bluma Guenter Soares ${ }^{\mathrm{ab}}$ and Fernando Gomes Souza, Jr (DD *ad
}

\begin{abstract}
The objective of this work is the development of a toxic gas detector/filter based on the production of porous polyaniline composites filled with magnetic nanoparticles. The composite produced was subjected to hydrogen sulfide gas as a preliminary test of its detection and sorption capacity, which were proven by gravimetric analysis. Analysis by light scattering and TEM indicated that magnetic nanoparticles with a size of approximately $5 \mathrm{~nm}$ were obtained through the proposed methodology. FTIR spectroscopy, UV-vis spectroscopy, TGA, and DSC were performed to prove the successful synthesis of the composite. To identify the specific properties of each constituent of the composite, the conductivity and magnetic force of the material were determined. The SEM results showed that the morphology was useful for the sorption process with the formation of pores in the polymer matrix, allowing the percolation of the gas for splicing by the nanoparticles. TGA, electrical conductivity, magnetic force, UVvis spectroscopy, and EDS analyses were also performed after the detection/sorption tests to demonstrate the functioning of the material.
\end{abstract}

\section{Introduction}

Since its discovery 180 years ago, ${ }^{1}$ polyaniline (PAni) has been reported in the literature as an intrinsically conductive polymer with great technological and scientific applicability due to its peculiar properties such as excellent thermal and environmental stability, ready availability and excellent electrical and optical properties. ${ }^{2,3}$ As a sensor, PAni has received significant attention ${ }^{4}$ since its different oxidation states allow changes in its optical properties when subjected to different acidic or alkaline analytes ${ }^{5}$ as well as its electrical properties. ${ }^{6}$

Given the excellent properties and advantages of PAni, the combination of this polymer with different materials has been the target of many studies to potentiate such properties as well as to form more efficient materials. ${ }^{7,8}$ The combination with inorganic materials, in particular oxides, has created a class of desirable polymer/metal materials for technological applications. ${ }^{9,10}$ The composites or hybrids of PAni and graphene or iron oxides, for example, have been the basis for the

\footnotetext{
${ }^{a}$ Macromolecules Institute: Professor Eloisa Mano, Technology Center-University City, av. Horácio Macedo, 2030, block J. Federal University of Rio de Janeiro, RJ, Brazil. E-mail: fernando_gomes@ima.ufrj.br

${ }^{b}$ Department of Metallurgical and Materials Engineering, COPPE, Technology CenterUniversity City, av. Horácio Macedo, 2030, bloco F. Federal University of Rio de Janeiro, RJ, Brazil

${ }^{c}$ Brazilian Center for Physical Research, CBPF, Rua Dr. Xavier Sigaud, 150 - Urca, Rio de Janeiro - RJ, Brazil

${ }^{d}$ Nanotechnology Engineering Program, COPPE, Technology Center-University City, av. Horácio Macedo, 2030, bloco F. Federal University of Rio de Janeiro, RJ, Brazil
}

development of polymers with better conductive and magnetic properties., ${ }^{8,11}$

Magnetic particles, especially iron oxide $\left(\mathrm{Fe}_{3} \mathrm{O}_{4}\right)$ nanoparticles, have been widely reported in the literature ${ }^{12}$ in applications in the area of cancer treatment with magnetic hyperthermia, water treatment, ${ }^{13,14}$ catalysts, ${ }^{15}$ and heavy metal adsorbers ${ }^{16-18}$ besides their use in the adsorbers of toxic gases. ${ }^{19}$ Their viability is due to their facile synthesis, good yields, and high sorption capacity as a result of the electrostatic interactions that contribute to excellent adhesion as well as the possibility of binding with macromolecules and functional groups on the surfaces of the magnetic particles. ${ }^{20}$

In this context, this work has the premise of obtaining PAni composites filled with iron oxide nanoparticles for the development of a toxic gas filter/sensor. The nanoparticles were previously obtained from a colloidal dispersion of polyvinyl alcohol (PVA) in order to obtain a suitable nanoscale size as well as promote a morphology conducive to the sorption of the composite. For the application of the proposed material, adsorption and detection tests were performed in the presence of hydrogen sulfide.

\section{Experimental section}

\section{Materials}

All chemicals were of analytical grade. Polyvinyl alcohol with a degree of hydrolysis of $89 \%$ (molecular weight, $M_{\mathrm{w}}$, of about $115.000 \mathrm{~g} \mathrm{~mol}^{-1}$, Sigma-Aldrich), ferrous chloride $\left(\mathrm{FeCl}_{2} \cdot 4 \mathrm{H}_{2} \mathrm{O}\right.$ (Sigma-Aldrich)), ferric chloride $\left(\mathrm{FeCl}_{3} \cdot 6 \mathrm{H}_{2} \mathrm{O}\right)$ (Sigma-Aldrich), 
sodium hydroxide $(\mathrm{NaOH})$ (Sigma-Aldrich), deionized water, aniline (Sigma-Aldrich), dodecylbenzene sulfonic acid (DBSA), and ammonium persulfate $\left(\left(\mathrm{NH}_{4}\right)_{2} \mathrm{~S}_{2} \mathrm{O}_{8}\right)$ (Sigma-Aldrich) were used without further purification.

\section{Synthesis of magnetic nanoparticles}

Magnetic nanoparticles were prepared following the methodology proposed by Souza Jr and co-workers. ${ }^{6}$ For the procedure, $1 \mathrm{~L}$ of an aqueous solution using different concentrations $(1 \%$, $2 \%$ and $5 \%$ ) of PVA deionized water was initially prepared with mechanical stirring at $50{ }^{\circ} \mathrm{C}$ for 1 hour.

PVA with different concentrations was used to identify the PVA/NPIO dispersion that formed the smallest size of nanoparticles.

$\mathrm{FeCl}_{2}$ and $\mathrm{FeCl}_{3}$, in a molar ratio $1: 2$, were then added to the $5 \%$ PVA solution. The solution was kept under stirring for 1 hour at room temperature to dissolve the added salts. $\mathrm{Fe}^{2+}$ and $\mathrm{Fe}^{3+}$ ions were then co-precipitated with $30 \mathrm{~mL}$ of 1 molar $\mathrm{NaOH}$ solution, added slowly, to obtain the magnetic nanoparticles. The magnetic nanoparticles were kept under mechanical agitation for 1 hour at room temperature. The obtained colloidal dispersion was centrifuged, with two cycles of 30 minutes each, at $4000 \mathrm{rpm}$ at room temperature. For comparative purposes, magnetic particles were synthesized by coprecipitating the $\mathrm{Fe}^{2+}$ and $\mathrm{Fe}^{3+}$ in an aqueous medium, in place of the PVA dispersion.

\section{Synthesis of PAni and magnetic nanoparticle composites}

The synthesis of PAni was performed following the procedure described elsewhere by our group. ${ }^{20}$ The synthesis of the composites was carried out by the in situ polymerization of PAni in the presence of the colloidal dispersion of nanoparticles. In the process, $100 \mathrm{~mL}$ of a DBSA solution, $1 \mathrm{~mol} \mathrm{~L}^{-1}$, was first prepared, which was maintained under mechanical stirring and room temperature for $1 \mathrm{~h}$. Then, the colloidal nanoparticle dispersion (NPOI), with a concentration of $20 \%$, previously measured by TGA, in which an amount of $0.53 \mathrm{mg}$ of magnetic nanoparticles was contained in $20 \mu \mathrm{L}$ of the dispersion, was added and kept in the shaking system for 1 hour. This was followed by the in situ polymerization of PAni, where aniline, $1 \mathrm{~mol} \mathrm{~L}^{-1}$, was added to the system and after 1 hour, $20 \mathrm{~mL}$ of an acid solution of the oxidizing agent, ammonium persulfate, $1 \mathrm{~mol} \mathrm{~L}^{-1}$, was slowly dripped into the reaction, which was maintained under mechanical stirring and at room temperature for 1 hour. After the reaction, the material was centrifuged with successive washings with alcohol, until the supernatant was colorless, to remove excess PVA to obtain the proposed morphology. The composite was obtained as a powder, PAni/ NPOI $20 \%$, and was dried at room temperature for 24 hours.

\section{Exposure of the material to toxic gases}

First, $0.5 \mathrm{~g}$ of each sample was inserted into the center of $7.00 \times$ $0.75(L \times D)$ plastic tubes of approximately $0.66 \mathrm{~g}$, which were discounted for gravimetric analysis.

The gas production and exposure system consisted of a Kipp pipette coupled to a $250 \mathrm{~mL}$ Erlenmeyer flask containing FeS and $\mathrm{HCl}$ in the $2: 1$ molar ratio and the system was heated to approximately $50{ }^{\circ} \mathrm{C}$. The plastic tubes containing the samples were connected to the Kipp pipette at one end and the other to a gas collection and identification system through a glass pipette. The collection and identification system consisted of a $250 \mathrm{~mL}$ round bottom flask with three openings. In the first opening, the $\mathrm{pH}$ meter electrode was connected to determine the variation of the gas concentration at the inlet and outlet; in the second opening was the plastic tube containing the sample, and the third end was sealed. The tube was weighed every 15 minutes, over $1 \mathrm{~h}$, to evaluate the mass variation during exposure.

\section{Characterization}

\section{Dynamic light scattering (DLS)}

The dispersions of magnetic nanoparticles in 1\%, $2 \%$ and $5 \%$ PVA were characterized using a particle size analyzer, the Zetasizer Nano ZS Malvern, in order to obtain the size range of the particles dispersed in the colloidal dispersion of PVA and their size distribution. The equipment had a detector at $173^{\circ}$ from the incident light beam, known as backscatter detection, patented technology known as NIBS using a refraction index of 1.700 , and a magnetite correspondent under room temperature conditions, with 20 scans in triplicate. For this analysis, 1 drop of the sample was diluted in $1 \mathrm{~mL}$ PVA solution, with the corresponding concentration, and inserted in a quartz bucket. The data were treated with a Qtiplot 0.9.8.6 program.

\section{Transmission electron microscopy - TEM}

The analyses were carried out at the Center for Structural Biology and Bioimaging - CENABIO, using an FEI Tecnai Spirit Electronic Transmitting Microscope with $120 \mathrm{kV}$ acceleration voltage and tungsten filament with a $2 \mathrm{k}$ Veleta Olympus digital camera. For analysis, $20 \mu \mathrm{L}$ of the magnetic nanoparticle dispersion (NPIO/PVA5\%) was diluted in $10 \mathrm{~mL}$ of 5\% PVA solution and dripped onto a formvar-coated copper grid with 200 mesh spacing.

\section{Thermogravimetric analysis and differential scanning calorimetry - TGA and DSC}

The tests were performed using a TA Instruments TGA Q500V6.7 Build 203 under an inert atmosphere of nitrogen (purge gas $-19.8 \mathrm{~mL} \mathrm{~min}^{-1}$ ), at temperatures between $30^{\circ} \mathrm{C}$ and $700{ }^{\circ} \mathrm{C}$ and a heating rate of $20^{\circ} \mathrm{C} \mathrm{min}{ }^{-1}$. In a typical test, $20 \mu \mathrm{L}$ of the sample, measured with the aid of an automatic micro-pipette, was used for the analysis. Tests were performed five times and the $95 \%$ confidence limits were calculated. The data were treated with a Qtiplot 0.9.8.6 program.

\section{Scanning electron microscopy and energy dispersive X-ray - SEM and EDX}

This analysis was carried out in partnership with the Mineral Technology Center (CETEM). For the tests, the samples in powder form were carbon-plated and placed in a JEOL model 
JSM-5610LV under a $20 \mathrm{kV}$ acceleration voltage with secondary electron detectors at magnifications of 100, 2000 and 5000 times.

\section{Atomic force microscopy}

The morphology (topography, amplitude, phase) of the surface of the samples was analyzed by AFM (HITACHI 5100N). On a mica surface, the sample was deposited in the solid state. The evaluation was performed intermittently at room temperature. Silicon tips of Atomic Force Microscopy HA_NC coupled to the cantilever of length $117 \mu \mathrm{m}$, with a resonance frequency of about $110 \mathrm{kHz}$ and a constant nominal force of $2 \mathrm{~N} \mathrm{~m}^{-1}$ were used. The images obtained were processed using the AFM5000 II Software.

\section{Electrical tests}

For the determination of electrical conductivity, the twoelectrode method was used according to the analyses already carried out in our research group at the Laboratory of Biopolymers and Sensors (LABIOS). For this, the samples were placed between the two electrodes. An electric voltage of $5 \mathrm{~V}$ was generated between these electrodes, which allowed the determination of the current that would cross the sample and the calculation of the conductivity of the materials. Conductivity tests were performed using a Keithley 6517B electrometer. The thicknesses of the samples were determined with the aid of a Digimess digital caliper. The electrical conductivity data, taken over two minutes of the experiment, were stored and used to calculate the resistivity.

\section{Magnetic force analysis}

Magnetic force tests were performed following a procedure reported elsewhere by our group. ${ }^{20}$ The experimental setup was composed of an analytical scale (Shimadzu AY-220), a voltage source (ICEL PS-4100), a digital multimeter ICEL MD-6450, a Gaussmeter GlobalMag TLMP-Hall-02, a home-made sample holder and a home-made electromagnet. The electromagnet was made with a solid $12 \mathrm{~cm}$ long iron core with a $1.6 \mathrm{~cm}$ diameter circular section, and 4200 turns of 18AWG copper wire. The data were treated with the Qtiplot 0.9.8.6 program.

\section{Magnetization analysis}

The magnetization tests were performed using a magnetometer from Quantum Design PPMS ${ }^{\circledR}$ VersaLab ${ }^{\mathrm{TM}}$. Tests were performed in partnership with the Magnetometric Laboratory of the Brazilian Center for Physical Research. The analyses were carried out at room temperature, with an oscillating magnetic field from $-30 \mathrm{kOe}$ to $+30 \mathrm{kOe}$. The samples were inserted in a plastic sample-holder with a mass of around $7.5 \mathrm{mg}$. The data were treated with the Qtiplot 0.9.8.6 program.

\section{The BET (Brunauer-Emmett-Teller) method - multimolecular adsorption analysis - ASAP}

To identify the sorption and desorption behavior of materials, we used the Adsorption Multimolecular Micromeritics ASAP 2010 analysis equipment with $\mathrm{N}_{2}$ gas 5.0 Analytical (White
Martins) and an $\mathrm{H}_{2}$ tablet saturation tube (White Martins). The magnetic particles, PAni and the composite magnetic nanoparticles (20\%), and PAni were used at treatment temperatures of $150{ }^{\circ} \mathrm{C}, 350{ }^{\circ} \mathrm{C}$, and $120^{\circ} \mathrm{C}$, respectively. The data were treated with the Qtiplot 0.9.8.6 program.

\section{Gravimetric analyses}

For gravimetric analyses, the samples were weighed on an analytical digital scale. They were then placed inside the system with gas production. Every 15 minutes, the samples were removed from the system, sealed and weighed to evaluate their mass variation until 60 minutes of exposure to the gas had been completed. Simultaneously, the $\mathrm{pH}$ of the system was measured to verify the amount of produced gas, in $\mathrm{ppm}$. The data were treated with the Qtiplot 0.9.8.6 program.

\section{Results and discussion}

\section{Colloidal dispersion of magnetic nanoparticles}

Dynamic light scattering (DLS). Fig. 1 shows the dynamic light scattering analysis to determine the size and distribution of the magnetic nanoparticles prepared using $1 \%, 2 \%$, and $5 \%$ PVA concentrations.

Particle size distribution analyses were performed to identify the concentration of PVA at which the smaller-sized magnetic nanoparticles were obtained. It was observed that for the colloidal dispersion of magnetic nanoparticles containing 5\% PVA, the smaller nanoparticle sizes between $2.32 \mathrm{~nm}$ and $4.84 \mathrm{~nm}$ were obtained with volumes of $13.50 \%$ and $14.03 \%$, respectively.

The decrease in the diameter of the $\mathrm{Fe}_{3} \mathrm{O}_{4}$ nanoparticles as a function of the increase in the amount of PVA was attributed to the increase in the dispersion stability, ${ }^{17}$ avoiding the agglomeration of nanoparticles. ${ }^{21,22}$ This effect had already been proven in the synthesis of nanoparticles of $\mathrm{Fe}_{3} \mathrm{O}_{4}$, with average diameters between 4 and $7 \mathrm{~nm}$ and with super magnetic properties. $^{23}$

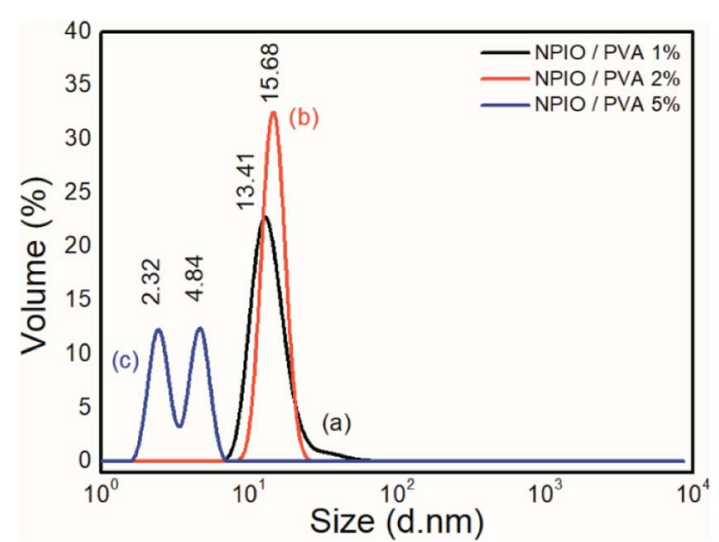

Fig. 1 Size distribution analysis of the colloidal dispersions of magnetic nanoparticles in PVA at 1\% (a), 2\% (b) and 5\% (c). 

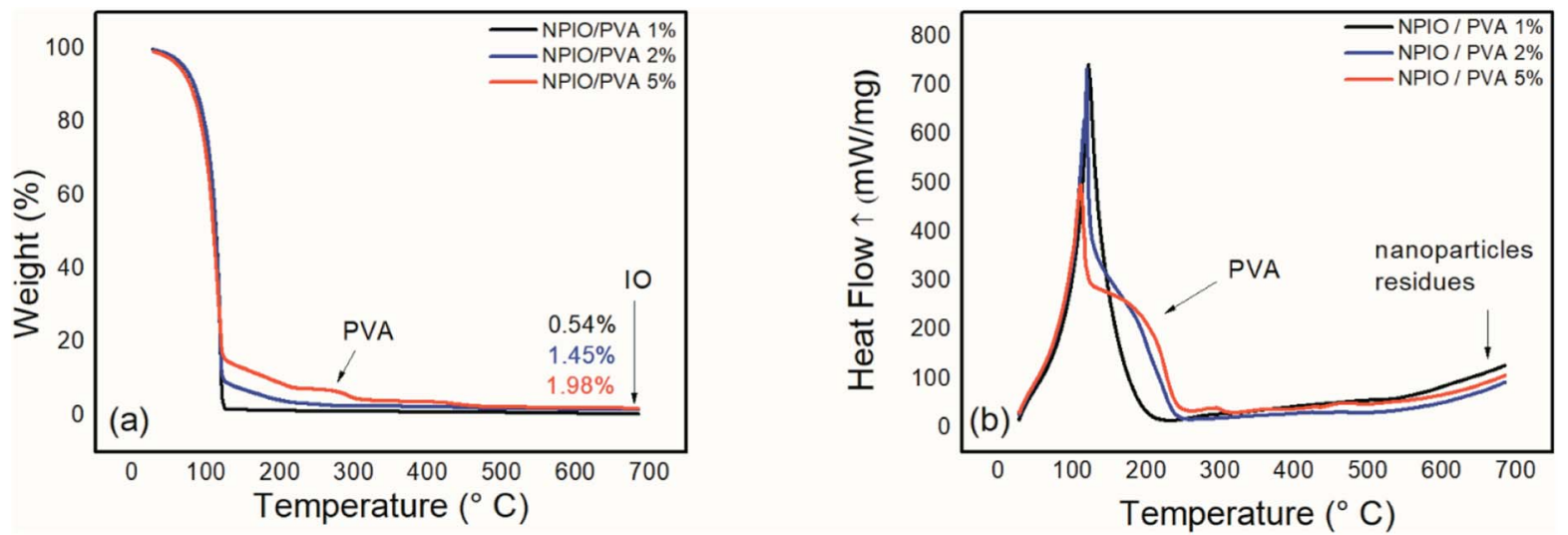

Fig. 2 Thermogravimetric analysis (a) and differential scanning calorimetry (b) for the colloidal dispersion of magnetic nanoparticles in PVA at 1\%, $2 \%$ and $5 \%$.

\section{Thermogravimetric analysis (TGA) and differential scanning calorimetry (DSC)}

Fig. 2(a) shows the thermogram of magnetic nanoparticle dispersions with $1 \%, 2 \%$, and $5 \%$ PVA.

Thermogravimetric analysis was conducted to identify the residual mass present in the colloidal dispersions of magnetic nanoparticles, as well as natural degradations of the samples. In all the dispersions, the first thermal degradation event was observed up to $126{ }^{\circ} \mathrm{C}$ and was attributed to water loss.

A second thermal degradation event was observed at around 266-307 ${ }^{\circ} \mathrm{C}$, which can be attributed to PVA decomposition. ${ }^{14,16} \mathrm{An}$ increase in mass loss in this temperature range was observed as the PVA concentration in the dispersion of the magnetic nanoparticles was increased, proving the increase in the polymeric material in the dispersion. Such a system containing $5 \%$ PVA in the dispersion prevents the decanting of magnetic nanoparticles, whose supernatant contains the most significant number of nanoparticles. For the dispersion containing 5\% PVA, a mass loss of approximately $3 \%$ was observed as being the most evident.

\section{Transmission electron microscopy - TEM}

Fig. 3 shows micrographs of the colloidal dispersion of magnetic nanoparticles in PVA at 5\%, with resolutions of 500, 200 , and $100 \mathrm{~nm}$.
The diameters of the $\mathrm{Fe}_{3} \mathrm{O}_{4}$ nanoparticles were confirmed by TEM. A good dispersion of nanoparticles with spherical morphologies was observed, thus confirming that the synthesis in the presence of $5 \%$ PVA prevented the agglomeration of the magnetic nanoparticles. ${ }^{20}$ Average diameters were found to be around (43.95 \pm 1.24$),(17.80 \pm 0.79)$ and $(6.95 \pm 1.06)$, which are considered as nanoparticles according to the literature, ${ }^{18,25}$ and favour the sorption process. ${ }^{15,21,26}$ Droplets that had nanoparticles of different sizes were also observed, but these were below $6.95 \mathrm{~nm}$, as shown in Fig. 3(c).

The third event, at around $300-450{ }^{\circ} \mathrm{C}$, was attributed to the elimination of all PVA on the surface of the nanoparticles of $\mathrm{Fe}_{3} \mathrm{O}_{4}$. The thermal degradation of the PVA ultimately proved the adsorption of the polymer by the surface of the inorganic material through the hydrogen bonds of the polar functional groups in the PVA and the iron oxide. ${ }^{23}$ Thus, the dispersion of magnetic nanoparticles in PVA at 5\% presented the best conditions for the proposed application. Therefore, this concentration was adopted for the synthesis of the composites since the concentration of PVA used prevented the agglomeration of the nanoparticles of $\mathrm{Fe}_{3} \mathrm{O}_{4}$ caused by the particleparticle interaction and its excellent surface energy. ${ }^{24}$

\section{PAni composites filled with magnetic nanoparticles}

Fourier-transform infrared spectroscopy - FTIR. The chemical structure of the materials, as well as the confirmation of the
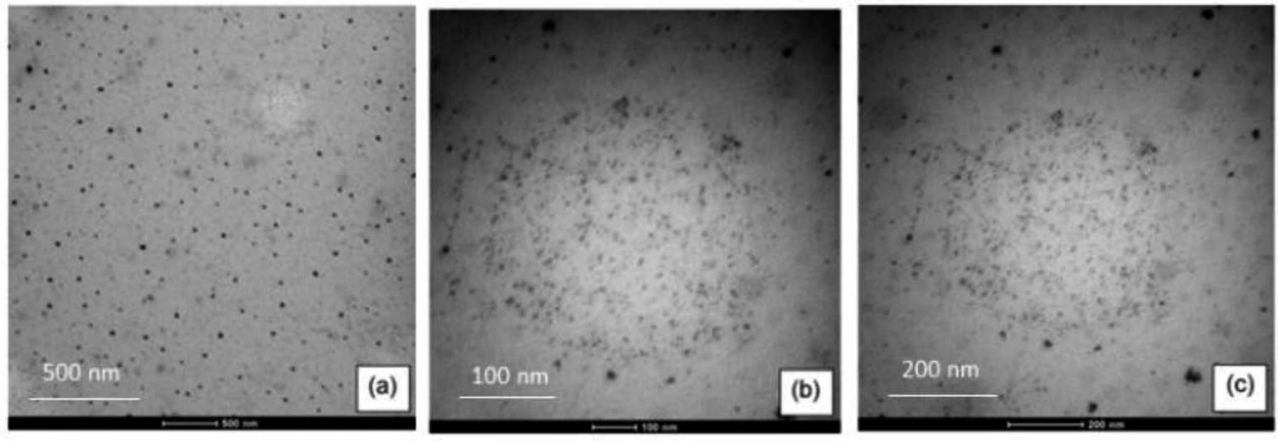

Fig. 3 TEM micrographs of the colloidal dispersion of 5\% PVA nanoparticles at (a) $500 \mathrm{~nm}$, (b) $200 \mathrm{~nm}$ and (c) $100 \mathrm{~nm}$ resolution. 
Table 1 Characteristic absorption bands of the prepared materials ${ }^{a}$

\begin{tabular}{lllllllll}
\hline & \multicolumn{7}{l}{ Wavelength $\left(\mathrm{cm}^{-1}\right)$} \\
\cline { 2 - 8 } Sample & $\mathrm{Fe}-\mathrm{O}$ & $\mathrm{Ar}$ & $\mathrm{Ar}-\mathrm{N}$ & $\mathrm{N}-\mathrm{B}-\mathrm{N}$ & $\mathrm{N}=\mathrm{Q}=\mathrm{N}$ & $\mathrm{H}_{2} \mathrm{O}$ & $\mathrm{OH}$ \\
\hline IO & $396 / 555$ & - & - & - & - & 1632 & 3295 \\
NPIO & 525 & - & - & - & - & 1632 & 3164 \\
PAni & - & 820 & 1115 & $1308 / 1491$ & 1576 & - & - \\
PAni/NPIO & $403 / 504$ & 823 & 1110 & 1420 & 1573 & 1648 & 3265
\end{tabular}

${ }^{a} \mathrm{Ar}$ is aromatic.

synthesis of the composite of PAni and magnetic nanoparticles, were investigated by Fourier transform infrared spectroscopy (FT-IR). Table 1 shows the absorptions of the insulating materials and the composite, with their respective wavelengths. Fig. 4 shows the absorption bands of each material.

Absorbances at wavelengths of $555 \mathrm{~cm}^{-1}$ and $396 \mathrm{~cm}^{-1}$ were attributed to $\mathrm{Fe}-\mathrm{O},{ }^{27}$ and absorptions at $3295 \mathrm{~cm}^{-1}$ and $1632 \mathrm{~cm}^{-1}$ were attributed to the vibrations of the $\mathrm{H}_{2} \mathrm{O}$ and $\mathrm{OH}$ groups, respectively, corresponding to the magnetic nanoparticles. ${ }^{28}$ These absorptions were also observed on the magnetic particles whose analyses were performed for comparative purposes. For pure PAni, absorptions were observed at around $1600 \mathrm{~cm}^{-1}$ and $1500 \mathrm{~cm}^{-1}$ and were attributed to the quinoid groups $(\mathrm{N}=\mathrm{Q}=\mathrm{N})$ and benzoids $(\mathrm{N}-\mathrm{B}-\mathrm{N})$, respectively, present in PAni. ${ }^{29}$ Other absorptions located around $1110 \mathrm{~cm}^{-1}$ and $820 \mathrm{~cm}^{-1}$ can be found in the structure of PAni and can be attributed to the vibrations of the aromatic rings and vibrations of the bonds between the nitrogen and the aromatic rings. ${ }^{30}$ For composite Fig. 4(c), absorptions were observed corresponding to both materials, thereby confirming the obtained PAni/NPIO 20\% composite.

\section{Morphological studies}

The morphological analyses by SEM and AFM were performed to identify the formation of pores in the polymer matrix in order
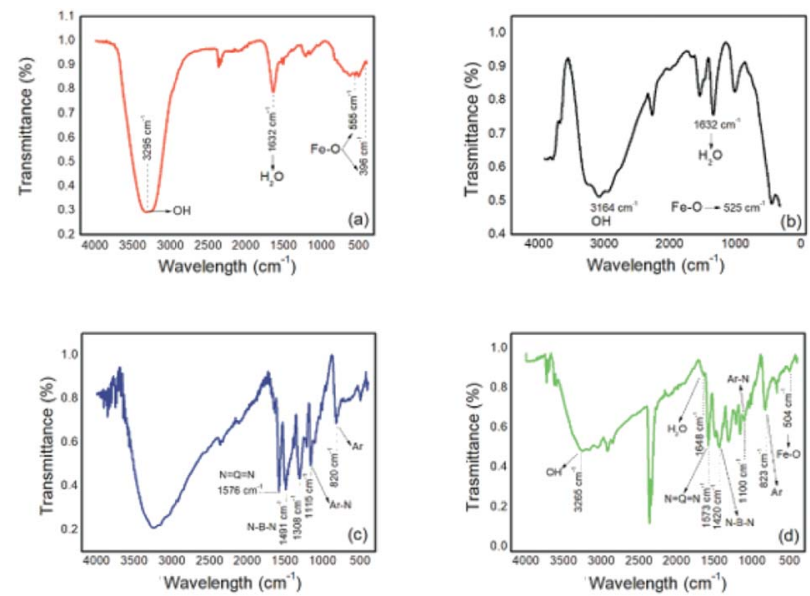

Fig. 4 Infrared spectra of (a) NPIO, (b) IO, (c) PAni and (d) PAni/NPIO $20 \%$.
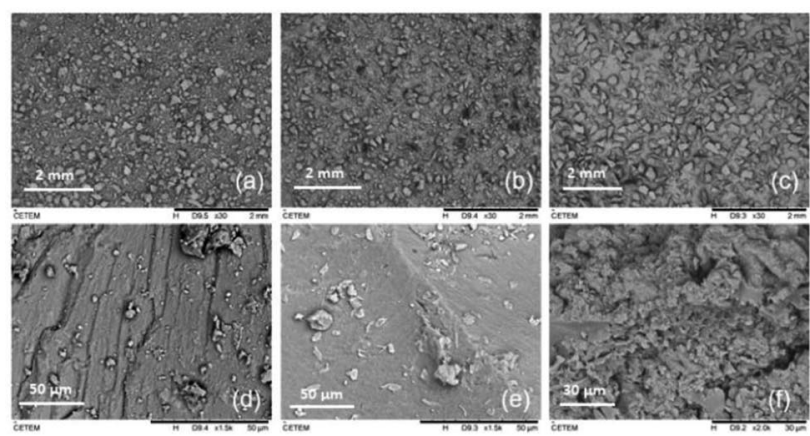

Fig. 5 SEM micrographs of (a) magnetic particles, (b) PAni and (c) PAni/NPIO 20\% composites with a magnification of 30 times. (d) Magnetic particles, (e) PAni and (f) PAni/NPIO 20\% with a magnification of 1500 and 2000 times.

to facilitate the transport of gas to the magnetic nanoparticles, allowing their sorption.

\section{Scanning electron microscopy - SEM}

Fig. 5 shows micrographs of the samples of iron oxide particles, the pure PAni, and the composite.

The SEM analysis could demonstrate the presence of a porous structure for the composite of PAni and the magnetic nanoparticles as compared with micrographs of the isolated materials, which had a smooth surface morphology.

The morphologies of the magnetic particles and PAni have already been proven in different studies, ${ }^{31}$ whose images showed materials with smooth surfaces. For PAni, ${ }^{32}$ in addition to its smooth structure, grooves were observed, which can be attributed to the presence of polymer ions. ${ }^{27}$

The morphology present in the composite, using 5\% PVA, confirmed the efficiency of the methodology proposed in this

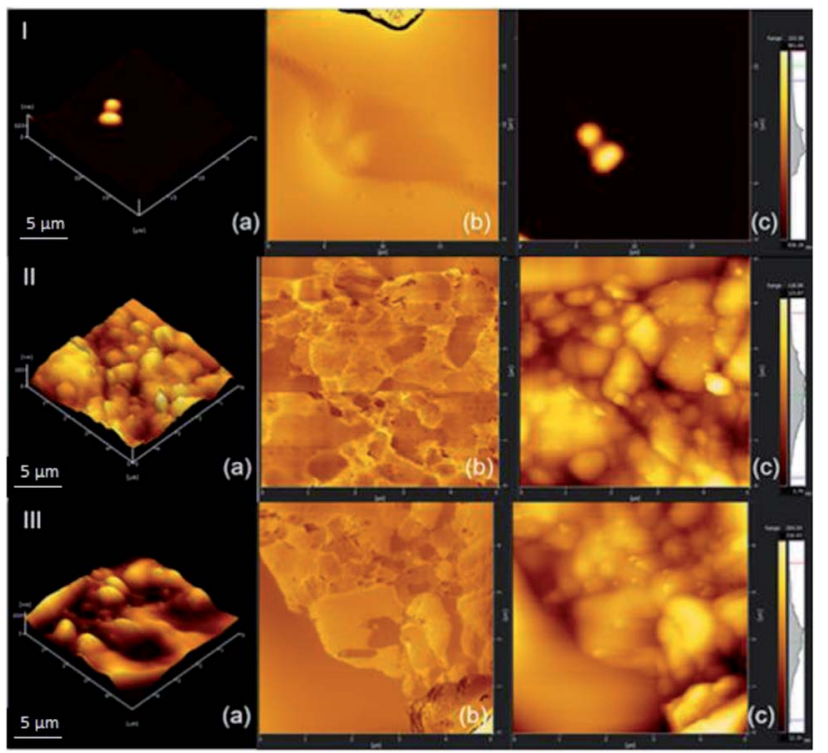

Fig. 6 AFM of the composite of (I) NPIO, (II) PAni and (III) PAni/NPIO 20\%: (a) 3D morphology, (b) phase analysis and (c) 2D topography. 


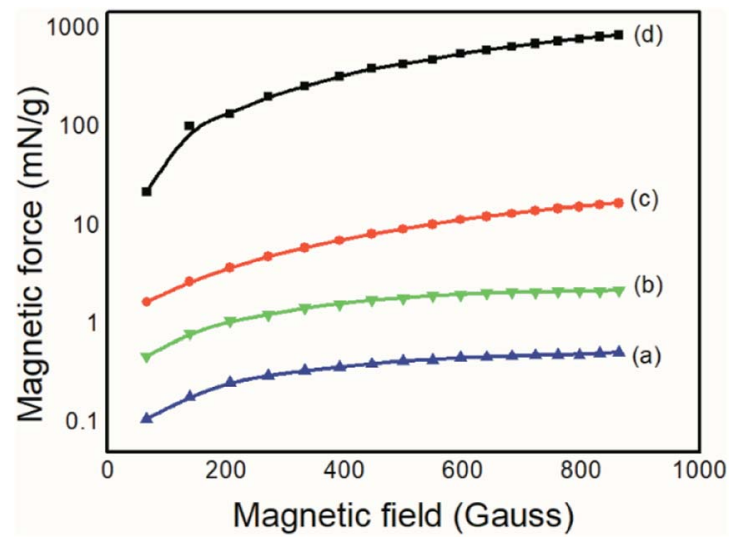

Fig. 7 Magnetic forces of (a) PAni, (b) PAni/NPIO 20\% composites, (c) NPIO, (d) 10.

work to obtain a composite with porous morphology. ${ }^{33-35}$ This morphology is useful for the detection of toxic gases by PAni, as well as for the adsorption of these contaminants.

\section{Atomic force microscopy - AFM}

Fig. 6 shows the composite morphological analysis by AFM. Fig. 6(a) shows a 3D map of the surface morphology over a small
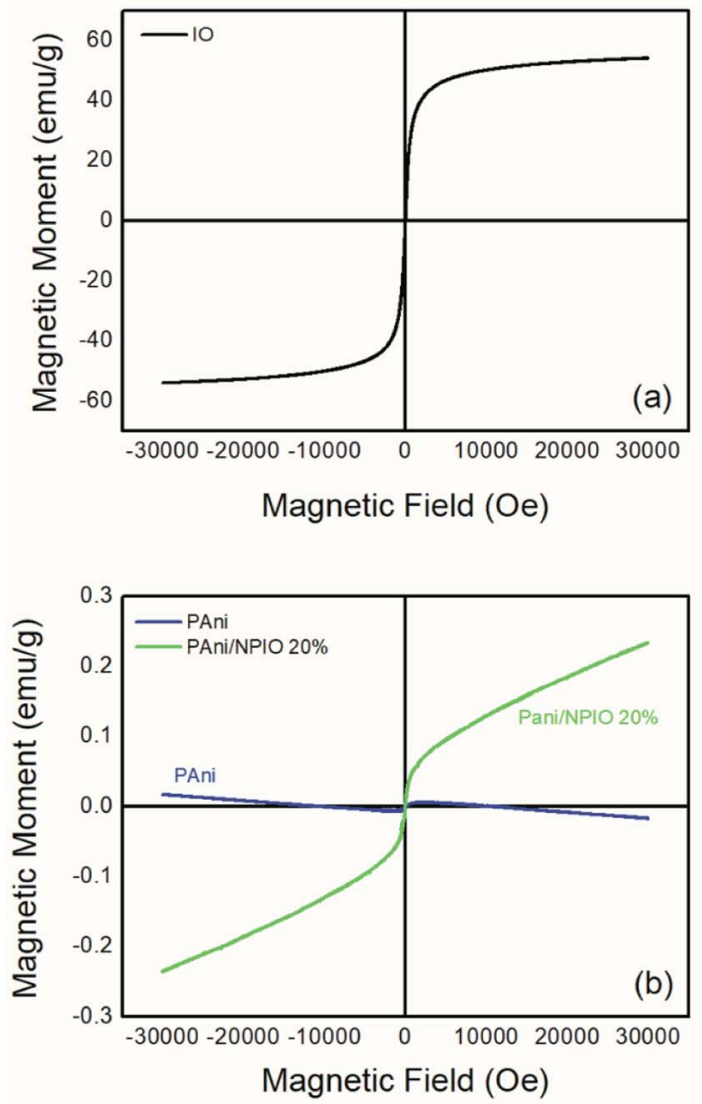

Fig. 8 Magnetization curves of (a) $1 \mathrm{O}$ and (b) pure PAni and the PAni/ NPIO 20\% composite. surface area $\left(5 \times 5 \mu \mathrm{m}^{2}\right)$ with the presence of valleys, which can be attributed to the formation of pores. This corroborates the results obtained by SEM, justifying the use of the proposed methodology for the application of the system in the sorption of gases.

The AFM technique is scarcely reported in the literature for the materials used in this work. However, studies on the preparation of composites containing PAni showed the presence of two phases, with the insertion of the polymer in the system. ${ }^{36,37}$ According to the literature, PAni presents a densely compacted morphology,,$^{33,38}$ which could be observed by the micrographs,
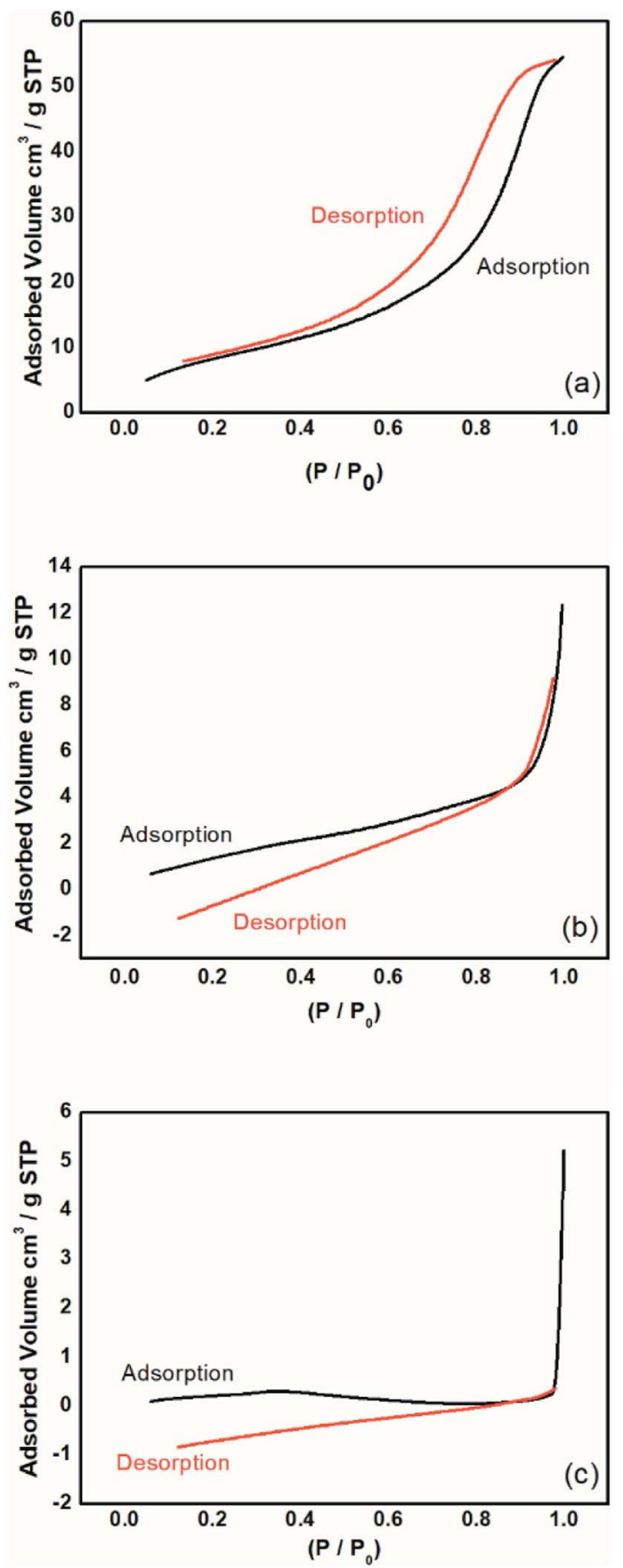

Fig. 9 Isotherms of adsorption and desorption analysis by the BET method for the (a) magnetic particles, (b) PAni, and (c) PAni/NPIO 20\%. 


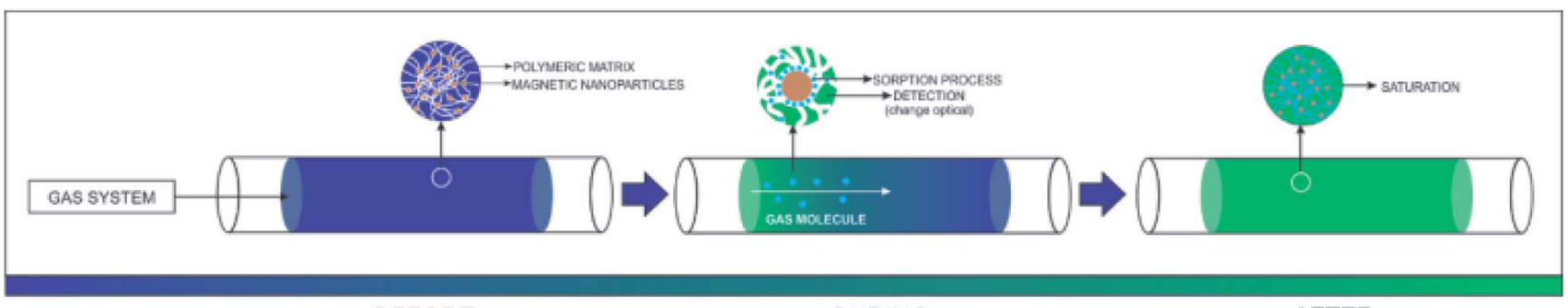

BEFORE

DURING

AFTER

Fig. 10 Representation of the device exposure to sulfide gas.

and which corroborates the results obtained by SEM. It is possible to observe in Fig. 6(c) the presence of 3 distinct colors, which can be attributed to PAni, on the surface, PVA dispersed in the medium, as well as magnetic nanoparticles, in the darkest parts, since polymers covered them.

\section{Magnetic properties studies}

Magnetic force analyses. In order to confirm the presence of magnetic nanoparticles, magnetic force and magnetization analyses were performed on each material. Fig. 7 shows the results of the magnetic force as a function of the applied magnetic field. Fig. 8 shows the results of the magnetization analysis.

The magnetite produced, for comparative purposes, presented a magnetic force of $867.71 \mathrm{mN} \mathrm{g}^{-1}$, which was above the expected value already reported in the literature. ${ }^{37}$ For the colloidal dispersion of magnetic nanoparticles, a value of 16.97 $\mathrm{N} \mathrm{g}^{-1}$ was obtained, below that of the magnetic particles. However, both the magnetic nanoparticles and the composite containing $20 \%$ of the magnetic nanoparticles had values of magnetic force that were 104 and 10 orders of magnitude higher than the pure PAni, respectively, thus proving the presence of the magnetic material, as expected.

\section{Magnetization analyses}

The magnetization describes how the material with magnetic properties reacts to an applied external magnetic field based on the alignment of their magnetic moment. ${ }^{39,40}$ Fig. 8 shows the magnetization curves of magnetization $M$, versus the applied magnetic field $\mathrm{H}$ for IO particles, pure PAni and the composite at room temperature under magnetic fields of $30 \mathrm{kOe}$.

In the IO particles, Fig. 8a, magnetization saturation occurred at a relatively low external field of approximately 3300 Gauss, with a value of $55.27 \mathrm{emu}^{-1}$ according to the literature. ${ }^{41}$ It is possible to observe zero coercivity and zero remanence on the magnetization curve, indicating the superparamagnetic behavior of the magnetite nanoparticles. ${ }^{42}$ For PAni, a non-magnetic moment aligned with the magnetic moment was observed (Fig. 8b), with 0 emu $\mathrm{g}^{-1}$ as expected. ${ }^{39}$ This is due to the presence of polyaniline. ${ }^{42}$ For the composite PAni/NPIO $20 \%$ the intermediate magnetization curve was observed as expected, indicating the presence of both materials in the composite.

\section{Gas exposure analysis}

BET method - multimolecular adsorption analysis - ASAP. Analyses of surface volume and pore size were performed on the materials via adsorption isotherms using the BET method. Fig. 9 shows the adsorption and desorption behaviors of the samples.

The magnetic particles had a surface area of $31.8 \mathrm{~m}^{2} \mathrm{~g}^{-1}$ with a calculated pore volume of $0.08 \mathrm{~cm}^{3} \mathrm{~g}^{-1}$ and average size of 116.16 A. PAni and PAni/NPIO 20\% presented surface area values equal to $6.48 \mathrm{~m}^{2} \mathrm{~g}^{-1}$ and $5.50 \mathrm{~m}^{2} \mathrm{~g}^{-1}$, with pore volume of $0.01 \mathrm{~cm}^{3} \mathrm{~g}^{-1}$ and $0.003 \mathrm{~cm}^{3} \mathrm{~g}^{-1}$ and diameters of $117.90 \AA$
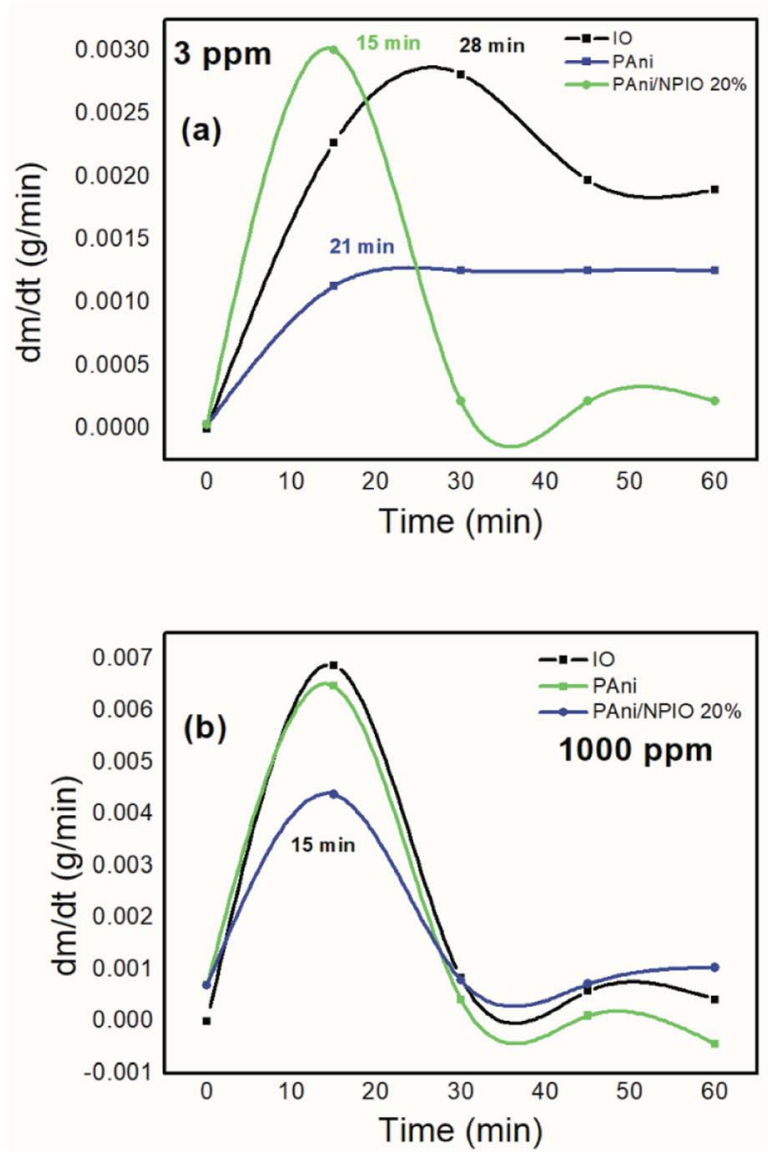

Fig. 11 First derivative of the gravimetric curves of the materials exposed to 3 (a) and $1000 \mathrm{ppm}$ (b) of $\mathrm{H}_{2} \mathrm{~S}$. 

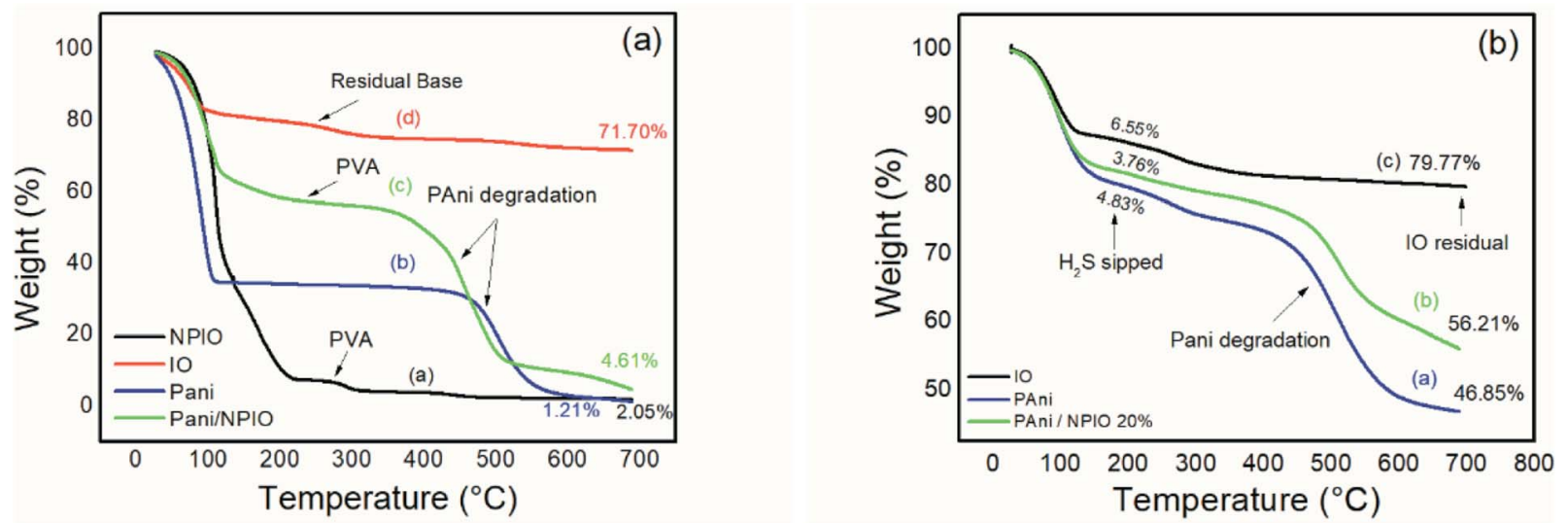

Fig. 12 Thermogram of materials (a) before and (b) after exposure to $\mathrm{H}_{2} \mathrm{~S}$ with a concentration of $3 \mathrm{ppm}$.

and $25.75 \AA$, respectively. Fig. 6 shows the adsorption and desorption tests of the materials in a $\mathrm{N}_{2}$ flow.

The observed adsorption isotherms are of type IV, according to IUPAC. ${ }^{43}$ Thus, the adsorbents possess pore sizes of diameters between 2 and $50 \mathrm{~nm}$, which are mesoporous. ${ }^{44}$ This behavior indicates that the relative pressure associated with the amount of gas in the system varies a little, the adsorbed volume increases substantially, and the process is reversible. ${ }^{39}$ This information confirms that the materials present gas adsorption characteristics and are applicable to the objective of this work.

It was also observed that the materials showed adsorption hysteresis, whose desorption does not coincide with the adsorption type $\mathrm{H} 1$, which indicates that there is a narrow distribution of relatively uniform pores with cylindrical shape. ${ }^{43}$ The presence of hysteresis also indicates that the materials, besides having pores, have pores with small sizes. The behavior identified by the isotherms proves the presence of pores, indicating the methodology efficiency proposed in this paper and corroborates the information obtained in the morphological analysis of the material.

Although the surface area, as well as the volume of gas adsorbed to the composites, has decreased, it should take into account the complex material system whose application is not limited only to adsorption by magnetic nanoparticles, but also includes the detection of gas by the PAni doping process, a gas fraction that will be retained in the polymer matrix.

\section{Proposed model for the detection/sorption/filtering process}

Fig. 10 shows the representation of the operation of the presented system. The inserted gas caused a change in the optical and electrical properties of PAni due to the doping process, as well as the electrical percolation of the polymer.

The PAni doping process is due to the formation of ions and counterions in the quinoid and benzoid units, ${ }^{6}$ forming bipolarons that are converted to polarons ${ }^{45}$ by the effect of the resonance of the electron. ${ }^{\mathbf{4 6 , 4 7}}$ The doping also produces a change in the PAni optical properties. More specifically, its conductive form is greenish, while the non-conducting one is blueish. ${ }^{\mathbf{4 8 , 4 9}}$ Concomitantly, the iron nanoparticles also participate in the adsorption process since the $\mathrm{H}_{2} \mathrm{~S}$ molecules react with the $\mathrm{OH}$ groups on the surface of the inorganic material, forming iron III sulfide $\left(\mathrm{Fe}_{2} \mathrm{~S}_{3}\right)$. The presence of moisture in the system also plays an important role. The $\mathrm{H}_{2} \mathrm{O}$ promotes the dissociation of $\mathrm{H}_{2} \mathrm{~S}$ into the sulfide ions $\left(\mathrm{HS}^{-}\right)$, which can replace the $\mathrm{OH}$ groups on the surface of the inorganic phase. ${ }^{50}$ Finally, iron oxides, especially magnetite, present an expressive density of $\mathrm{O}^{2-}$ in their structure. After the consumption of the $\mathrm{OH}$ groups, the $\mathrm{O}^{2-}$ species interacted with the $\mathrm{H}_{2} \mathrm{~S}$ molecules, reducing the Fe III complexes, leading to the formation of $\mathrm{SO}_{2} \cdot{ }^{51,52}$ Thus, multiple mechanisms can act together in the proposed material, improving, in theory, the efficiency of the capture of toxic vapors.

\section{Gravimetric analyses}

The gravimetric analyses were performed, aiming to determine the mass variation of the materials over the experiment time. Fig. 11 shows the first derivative of the gravimetric curves during exposure to $\mathrm{H}_{2} \mathrm{~S}$ gas at 3 and 1000 ppm for 60 minutes.

The maximum sorption rate indicated that in the first 15 minutes, for the $3 \mathrm{ppm}$ gas concentration, the composite PANI/ NPIO 20\% showed more significant adsorption in comparison with pure PANI and IO, proving the efficiency of the material. On the other hand, for the tests at $1000 \mathrm{ppm}$, all the materials presented the same saturation time of around 15 minutes. This behavior is due to the elevated concentration of the gas, which quickly saturated all the systems. However, the established levels for humans to be exposed without causing damage is below 10 ppm. ${ }^{53,54}$

\section{Thermogravimetric studies}

Fig. 12 shows the TGA of the materials before exposure (a) and after exposure (b) to $\mathrm{H}_{2} \mathrm{~S}$.

Thermogravimetric analyses were performed on the insulating materials and composites before and after exposure to hydrogen sulfide gas. Fig. 12a shows pre-exposure thermal degradations between $100{ }^{\circ} \mathrm{C}$ and $200{ }^{\circ} \mathrm{C}$ for all materials, most clearly for pure PAni and for the dispersion of magnetic nanoparticles in PVA, which can be attributed to water loss and some 


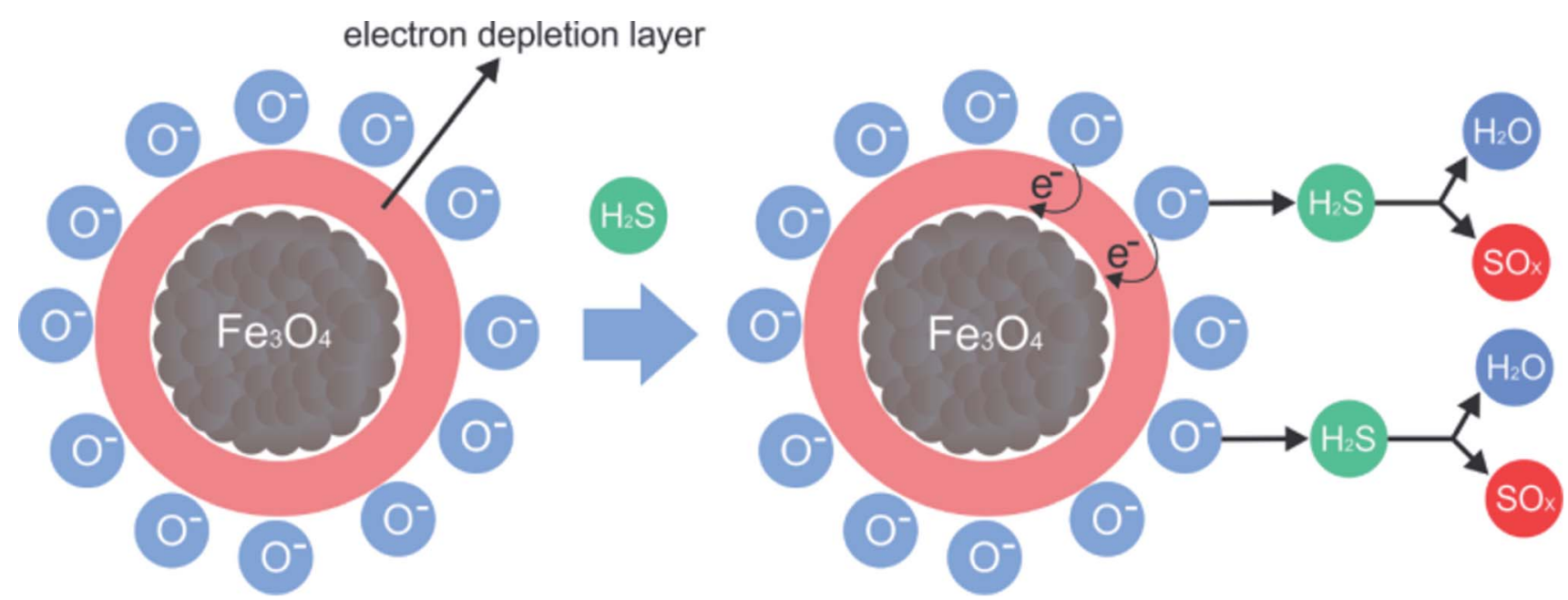

Fig. 13 Proposed mechanism for the $\mathrm{H}_{2} \mathrm{~S}$ adsorption process using magnetic nanoparticles and forming $\mathrm{SO}_{x}$ and $\mathrm{H}_{2} \mathrm{~S}$ species; adapted from Chen et al. 2017. ${ }^{17}$

components in the pure PAni, ${ }^{35}$ and loss of water for the dispersion of NPIO.

The second thermal decomposition, after $100^{\circ} \mathrm{C}$ extending up to $500{ }^{\circ} \mathrm{C}$, was observed in all materials except for pure PAni and the composite. Degradations between $200{ }^{\circ} \mathrm{C}$ and $300{ }^{\circ} \mathrm{C}$ were attributed to the presence of the dopant; ${ }^{55}$ its absence indicates its withdrawal altogether, or a good part of it, confirming the efficiency of the doping. This process of doping is essential for the application since the acid for the synthesis of the polymer and the acid gas have sulfur in their composition. The third thermal decomposition for the PAni and the composite was attributed to the degradation of the polymer chains. ${ }^{56}$

For the IO particles, NPIO dispersion and the composite, thermal degradations were observed before $200{ }^{\circ} \mathrm{C}$, which can be attributed to the water decomposition and groups corresponding to PVA. A second thermal degradation event, around $200{ }^{\circ} \mathrm{C}$, and $300{ }^{\circ} \mathrm{C}$ were also observed and correspond to the dehydration of -OH groups in the polymer chains of PVA present in the dispersion of magnetic nanoparticles. The third thermal decomposition for the NPIO after $300^{\circ} \mathrm{C}$ was attributed to the degradation of the PVA on the surface of the magnetic nanoparticles. ${ }^{57}$

PAni and the composite presented a thermal decomposition from $200{ }^{\circ} \mathrm{C}$ to $300^{\circ} \mathrm{C}$, which was attributed to the doping of the polymer and the composite by the acid gas. In comparison to the analogous non-tested materials, for the materials exposed in this process, when $\mathrm{Fe}_{3} \mathrm{O}_{4}$ is in contact with air, oxygen molecules are absorbed on its surface, forming chemically active species with negative charges and capturing electrons from the conduction layer, which forms an electron-depleting layer (Fig. 13).

When the gas comes into contact with magnetic nanoparticles, negatively charged oxygen molecules form species of sulfur oxide and water. ${ }^{51}$

\section{Optical properties studies - ultraviolet spectroscopy in the visible region (UV-vis)}

To identify changes in the optical properties of PAni, UV-vis analyses of the PAni and composite were performed before and after their exposure. Fig. 14 shows the corresponding absorptions of each material.

Absorbances at $577 \mathrm{~nm}$ and $616 \mathrm{~nm}$ were observed for the composites and PAni. They correspond to the $\pi-\pi^{*}$ transitions, of the non-conductive PAni. ${ }^{57}$ The shift to longer wavelengths indicates that PAni was obtained in its doped state. ${ }^{29}$ The observed shifts in the absorptions of PAni and the composite confirmed the changes in the optical properties of the materials, proving that they can be used as sensors for the detection of $\mathrm{H}_{2} \mathrm{~S}$ gas.

\section{Electrical resistivity}

Table 2 shows the values of the electrical resistivity of the materials before and after exposure to hydrogen sulfide.

For pure PAni and the PAni/NPIO 20\% composite, in its insulating form, emeraldine base, a high electrical resistivity value was observed, equal to $(9.8 \pm 0.6) \times 10^{8} \Omega \mathrm{cm}$ and $(9.8 \pm$ $0.5) \times 10^{8} \Omega \mathrm{cm}$, respectively. This high electrical resistivity value for PAni in its nonconductive form is attributed to the removal of charge carriers, called ions and counter ions, which reduce

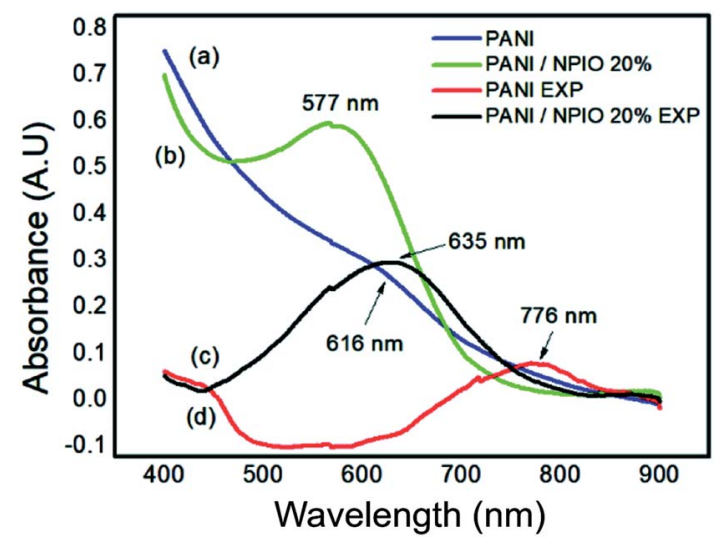

Fig. 14 UV-vis spectra of (a) pure PAni and (b) the composite before exposure and (c) pure PAni and (d) composite after exposure to $\mathrm{H}_{2} \mathrm{~S}$. 
Table 2 Electrical resistivities of the magnetic particles of IO, PAni and the PAni/NPIO 20\% composite

\begin{tabular}{lll}
\hline & \multicolumn{2}{l}{ Electrical resistivity $(\Omega \mathrm{cm})$} \\
\cline { 2 - 3 } Sample & Before exposure & After exposure \\
\hline IO particles & $1.0 \pm 0.1 \times 10^{9}$ & $5.5 \pm 0.7 \times 10^{6}$ \\
PAni & $9.8 \pm 0.6 \times 10^{8}$ & $2.7 \pm 0.4 \times 10^{6}$ \\
NPIO/PAni & $9.8 \pm 0.5 \times 10^{8}$ & $2.8 \pm 0.4 \times 10^{6}$
\end{tabular}
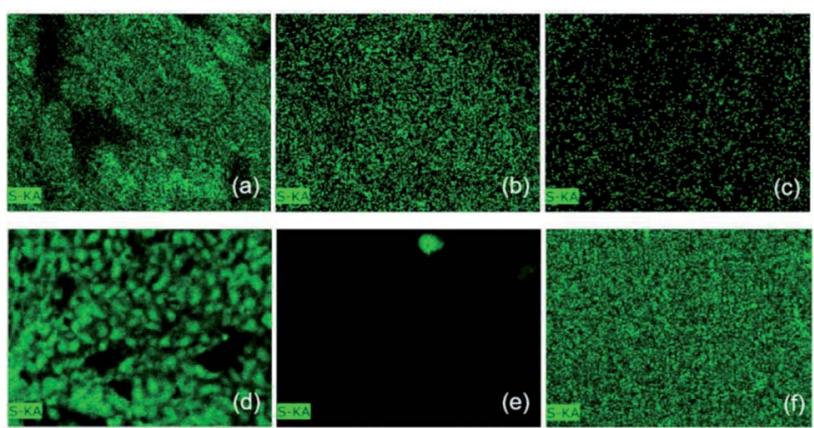

Fig. 15 Elementary mapping of the $S$ in (a) magnetic particles, (b) pure PAni and (c) the PAni/NPIO 20\% composite before exposure, and (d) magnetic particles, (e) pure PAni and (f) PAni/NPIO 20\% composite after exposure to $\mathrm{H}_{2} \mathrm{~S}$ gas with a concentration of $3 \mathrm{ppm}$.

carriers were removed, the electrical resistivity valu increased. ${ }^{46}$ The iron oxide particles presented electrical resistivity equal to $(1.0 \pm 0.1) \times 10^{9} \Omega \mathrm{cm}$. The value of the electrical resistivity can be attributed to the existence of lattice disorder or vacancies that affect the conduction mechanism.

After exposure to hydrogen sulfide, an increase in the electrical resistivity value of the magnetic particles was observed. Semiconductor metal oxides in gases tend to undergo changes in their electrical resistivities ${ }^{37,50}$ due to the reduction of these gases by the oxidative interactions with chemically adsorbed, negatively charged oxygen. When the interactions occur between the oxygen of the iron oxide and the acid gas, the $\mathrm{Fe}^{2+}$ ions are oxidized to $\mathrm{Fe}^{3+}$, forming a layer of electron depletion and promoting the appearance of holes in the structure of the solid, which prevents the transport of electrons due to the absence of charge carriers, thus the electrical resistivity of the material increases.

After exposure to the hydrogen sulfide gas, a decrease in the electrical resistivity value of pure PAni was observed from $(9.8 \pm$ $0.6) \times 10^{8} \Omega \mathrm{cm}$ to $(2.7 \pm 0.4) \times 10^{6} \Omega \mathrm{cm}$. For the composites of PAni/NPIO, a decrease in the resistivity value was observed, (9.8 $\pm 0.5) \times 10^{8}$ to $(2.8 \pm 0.4) \times 10^{6}$. Such a drastic change in the electrical properties of the polymer is attributed to the doping process in the PAni structure, caused by the acid gas.

Table 3 Elemental composition of materials before and after exposure to $\mathrm{H}_{2} \mathrm{~S}$ gas

Mass\% (normalized) before exposure

\begin{tabular}{llll}
\hline & OF & PAni & PAni/NPIO \\
\hline Nitrogen & 0.00 & 90.54 & 73.30 \\
Iron & 95.35 & 0.00 & 13.22 \\
Sulfur & 4.65 & 9.46 & 13.48 \\
\hline
\end{tabular}

Mass\% (normalized) after exposure

\begin{tabular}{llll}
\hline & OF & PAni & PAni/NPIO \\
\hline Nitrogen & 10.57 & 69.74 & 37.79 \\
Iron & 38.25 & 0.05 & 5.40 \\
Sulfur & 51.18 & 30.21 & 56.87 \\
Gain\% sulfur & 46.53 & 20.75 & 43.39
\end{tabular}

composite with characteristics for the detection and adsorption of $\mathrm{H}_{2} \mathrm{~S}$ gas was obtained by joining the electrical/optical properties of PAni and magnetic nanoparticles. The nanoparticles were characterized by light scattering and TEM. The porosity in the polymer matrix was determined by adsorption and desorption isotherms and were analyzed by SEM and AFM. Through the testing of the composite exposure to $\mathrm{H}_{2} \mathrm{~S}$ gas, in extreme concentrations, it was possible to identify a good response of the material at low gas concentrations and a lower exposure time below 15 minutes. The response of the material 
to the gas was verified by employing gravimetric analysis, thermogravimetric, optical, and electrical analyses, with the alteration of the intrinsic properties of PAni. The elemental analysis of the material before and after the exposure, indicated a dramatic increase in sulfur in comparison to the non-exposed PAni, which can be attributed to the doping process of the conducting polymer and sorption by the magnetic nanoparticles. At $\mathrm{H}_{2} \mathrm{~S}$ concentration of $3 \mathrm{ppm}$, the maximum sorption on the derivative showed an increase for the composite, confirming its sorption/detection capacity. The analyses before and after exposure to gas proved that both materials had a positive influence on the application proposed herein. This work has shown that the proposed material has the potential for application in the detection and sorption of toxic gases, especially $\mathrm{H}_{2} \mathrm{~S}$, which was the study gas for the development of the sensor.

\section{Conflicts of interest}

There are no conflicts to declare.

\section{Acknowledgements}

This work was carried out with CNPq (304500/2019-4) support, National Council for Scientific and Technological Development - Brazil, to the Coordination of Personal Improvement of Higher Education (CAPES - Project fund 001) and to Antonieta Middea and Mineral Technology Center, (CETEM).

\section{Notes and references}

1 G. Ćirić-Marjanović, Synth. Met., 2013, 177, 1-47.

2 K. R. Reddy, K. P. Lee and A. I. Gopalan, Colloids Surf., A, 2008, 320, 49-56.

3 M. T. Ramesan and T. Sampreeth, J. Mater. Sci.: Mater. Electron., 2018, 29, 4301-4311.

4 F. G. Souza, G. E. Oliveira, T. Anzai, P. Richa, T. Cosme, M. Nele, C. H. M. Rodrigues, B. G. Soares and J. C. Pinto, Macromol. Mater. Eng., 2009, 294, 739-748.

5 T. M. de Almeida, F. da S. Maranhão, F. V. de Carvalho, A. Middea, J. R. de Araujo and F. G. de S. Júnior, Macromol. Symp., 2018, 381, 1800111.

6 I. M. Ali, A. M. Shano and N. A. Bakr, J. Mater. Sci.: Mater. Electron., 2018, 29, 11208-11214.

7 J. H. Kim, F. F. Fang, H. J. Choi and Y. Seo, Mater. Lett., 2008, 62, 2897-2899.

8 A. Singh, N. P. Singh and R. A. Singh, Bull. Mater. Sci., 2011, 34, 1017.

9 R. Han, L. Zou, X. Zhao, Y. Xu, F. Xu, Y. Li and Y. Wang, Chem. Eng. J., 2009, 149, 123-131.

10 X. Yan, J. Chen, J. Yang, Q. Xue and P. Miele, ACS Appl. Mater. Interfaces, 2010, 2, 2521-2529.

11 M. Bhaumik, H. J. Choi, R. I. McCrindle and A. Maity, J. Colloid Interface Sci., 2014, 425, 75-82.

12 A. S. RezazadehNochehdehi, M. Sandri and A. Mohammadzadeh, in World Congress on Medical Physics and Biomedical Engineering, June 7-12, 2015, Toronto,
Canada, ed. D. A. Jaffray, Springer International Publishing, Cham, 2015, pp. 827-827.

13 F. Chen, S. Xie, X. Huang and X. Qiu, J. Hazard. Mater., 2017, 322, 152-162.

14 A. E. Chávez-Guajardo, J. C. Medina-Llamas, L. Maqueira, C. A. Andrade, K. G. Alves and C. P. de Melo, Chem. Eng. J., 2015, 281, 826-836.

15 G. Darabdhara, P. K. Boruah, N. Hussain, P. Borthakur, B. Sharma, P. Sengupta and M. R. Das, Colloids Surf., A, 2017, 516, 161-170.

16 M. J. Jafari, R. Zendehdel, A. Rafieepour, M. Nakhaei Pour, H. Irvani and S. Khodakarim, Int. J. Environ. Sci. Technol., 2020, 17, 187-194.

17 F. G. Souza, A. C. Ferreira, A. Varela, G. E. Oliveira, F. Machado, E. D. Pereira, E. Fernandes, J. C. Pinto and M. Nele, Polym. Test., 2013, 32, 1466-1471.

18 E. D. Pereira, F. G. Souza, C. I. Santana, D. Q. Soares, A. S. Lemos and L. R. Menezes, Polym. Eng. Sci., 2013, 53, 2308-2317.

19 H. Pardoe, W. Chua-anusorn, T. G. St. Pierre and J. Dobson, J. Magn. Magn. Mater., 2001, 225, 41-46.

20 I. Karimzadeh, M. Aghazadeh, A. Dalvand, T. Doroudi, P. H. Kolivand, M. R. Ganjali and P. Norouzi, Mater. Res. Innovations, 2019, 23, 1-8.

21 W. Wu, Z. Wu, T. Yu, C. Jiang and W.-S. Kim, Sci. Technol. Adv. Mater., 2015, 16, 023501.

22 T. L. Nguyen, T. R. Nizamov, M. A. Abakumov, I. V. Shchetinin, A. G. Savchenko and A. G. Majouga, Bull. Russ. Acad. Sci.: Phys., 2018, 82, 1214-1221.

23 S. Kayal and R. V. Ramanujan, Mater. Sci. Eng., C, 2010, 30, 484-490.

24 P. Arévalo, J. Isasi, A. C. Caballero, J. F. Marco and F. MartínHernández, Ceram. Int., 2017, 43, 10333-10340.

25 F. G. de Souza, J. A. Marins, J. C. Pinto, G. E. de Oliveira, C. M. Rodrigues and L. M. T. R. Lima, J. Mater. Sci., 2010, 45, 5012-5021.

26 H. Gavilán, O. Posth, L. K. Bogart, U. Steinhoff, L. Gutiérrez and M. P. Morales, Acta Mater., 2017, 125, 416-424.

27 F. Buazar, M. H. Baghlani-Nejazd, M. Badri, M. Kashisaz, A. Khaledi-Nasab and F. Kroushawi, Starch Staerke, 2017, 68(7-8), 796-804.

28 H. Wang, X. Yan and G. Piao, Electrochim. Acta, 2017, 231, 264-271.

29 G. Rizzo, A. Arena, N. Donato, M. Latino, G. Saitta, A. Bonavita and G. Neri, Thin Solid Films, 2010, 518, 71337137.

30 R. B. Patil, A. A. Jatratkar, R. S. Devan, Y.-R. Ma, R. K. Puri, V. Puri and J. B. Yadav, Appl. Surf. Sci., 2015, 327, 201-204.

31 R. Ullah, S. Bilal, A.-H. A. Shah, K. Ali and F. Alakhras, Synth. Met., 2016, 222, 162-169.

32 M. C. Arenas, G. Sánchez, O. Martínez-Álvarez and V. M. Castaño, Compos. B Eng., 2014, 56, 857-861.

33 R. Arora, U. K. Mandal, P. Sharma and A. Srivastav, Procedia Mater. Sci., 2014, 6, 238-243.

34 C. Steffens, A. Manzoli, J. E. Oliveira, F. L. Leite, D. S. Correa and P. S. P. Herrmann, Sens. Actuators, B, 2014, 191, 643649. 
35 H. Gu, Y. Huang, X. Zhang, Q. Wang, J. Zhu, L. Shao, N. Haldolaarachchige, D. P. Young, S. Wei and Z. Guo, Polymer, 2012, 53, 801-809.

36 F. Zhao, B. Zhang and L. Feng, Mater. Lett., 2012, 68, 112114.

37 G. D. Prasanna, H. S. Jayanna, A. R. Lamani and S. Dash, Synth. Met., 2011, 161, 2306-2311.

38 F. Ahmed, S. Kumar, N. Arshi, M. S. Anwar, L. Su-Yeon, G.-S. Kil, D.-W. Park, B. H. Koo and C. G. Lee, Thin Solid Films, 2011, 519, 8375-8378.

39 G. Ren, L. Yang, Z. Zhang, B. Zhong, X. Yang and X. Wang, J. Alloys Compd., 2017, 710, 875-879.

40 I. Fratoddi, I. Venditti, C. Cametti and M. V. Russo, Sens. Actuators, B, 2015, 220, 534-548.

41 N. Chaudhary, A. Singh, D. K. Aswal, P. Jha, S. Samanta, A. K. Chauhan, A. K. Debnath, S. Acharya, K. Shah, K. P. Muthe and S. C. Gadkari, Radiat. Phys. Chem., 2018, 153, 131-139.

42 D. S. Dhawale, A. Vinu and C. D. Lokhande, Electrochim. Acta, 2011, 56, 9482-9487.

43 J. Scotto, M. I. Florit and D. Posadas, Electrochim. Acta, 2018, 268, 187-194.

44 J. A. Arcibar-Orozco, R. Wallace, J. K. Mitchell and T. J. Bandosz, Langmuir, 2015, 31, 2730-2742.

45 Y. Huang, W. Chen, S. Zhang, Z. Kuang, D. Ao, N. R. Alkurd, W. Zhou, W. Liu, W. Shen and Z. Li, Appl. Surf. Sci., 2015, 351, 1025-1033.
46 Z. Li, Y. Huang, S. Zhang, W. Chen, Z. Kuang, D. Ao, W. Liu and Y. Fu, J. Hazard. Mater., 2015, 300, 167-174.

47 R. C. Ropp, in Encyclopedia of the Alkaline Earth Compounds, ed. R. C. Ropp, Elsevier, Amsterdam, 2013, pp. 105-197.

48 M. Das and D. Sarkar, J. Mater. Sci.: Mater. Electron., 2016, 27, 4109-4119.

49 C.-H. Chen, C.-J. Ko, C.-H. Chuang, C.-F. Mao, W.-T. Liao and C.-D. Hsieh, J. Polym. Res., 2016, 24, 10.

50 S. S. Umare, B. H. Shambharkar and R. S. Ningthoujam, Synth. Met., 2010, 160, 1815-1821.

51 J. Bhadra, N. K. Madi, N. J. Al-Thani and M. A. Al-Maadeed, Synth. Met., 2014, 191, 126-134.

52 M. R. dos Santos, J. J. Alcaraz-Espinoza, M. M. da Costa and H. P. de Oliveira, Mater. Sci. Eng., C, 2018, 89, 33-40.

53 L. Horta-Romarís, M.-J. Abad, M. V. González-Rodríguez, A. Lasagabáster, P. Costa and S. Lanceros-Méndez, Mater. Des., 2017, 114, 288-296.

54 Y. Long, Z. Chen, J. L. Duvail, Z. Zhang and M. Wan, Phys. B, 2005, 370, 121-130.

55 P. Prieto, P. Ruiz, I. J. Ferrer, J. de la Figuera and J. F. Marco, J. Alloys Compd., 2015, 636, 150-155.

56 K. Wetchakun, T. Samerjai, N. Tamaekong, C. Liewhiran, C. Siriwong, V. Kruefu, A. Wisitsoraat, A. Tuantranont and S. Phanichphant, Sens. Actuators, B, 2011, 160, 580-591.

57 B. Li, X. Weng, G. Wu, Y. Zhang, X. Lv and G. Gu, J. Saudi Chem. Soc., 2017, 21, 466-472. 